\title{
DUST IN NEARBY GROUPS OF GALAXIES?
}

\author{
M. Girardi, ${ }^{1}$ G. Giuricin, ${ }^{2}$ F. Mardirossian, ${ }^{2}$ M. Mezzetti $^{2}$ \\ ${ }^{1}$ S.I.S.S.A., Strada Costiera 11, 34014 Trieste, Italy \\ ${ }^{2}$ Department of Astronomy, via G.B. Tiepolo 11, 34131 Trieste, Italy
}

\begin{abstract}
The excess of higher redshift galaxies relative to the brightest member, found by several authors in poor, loose groups, is found to be present also in nearby, small groups identified by Geller and Huchra (1983) in the Center for Astrophysics Survey. This effect is explained as being due to the presence of diffuse intragroup dust together with an early evolutionary stage of nearby galaxy groups. An expected negative correlation between color excesses of galaxies and the velocity relative to the brightest member is found to be highly significant for both B-V and U-B colors. The optical depth produced by dust in groups turns out to be on the order of one, with a density of about $10^{-29} \mathrm{~g} / \mathrm{cm}^{3}$.
\end{abstract}

\section{INTRODUCTION}

Asymmetries in the redshift distribution (i.e., an excess of positive redshifts) of group members with respect to the redshift of the first-ranked spiral galaxy of many groups have often been reported in the literature and gave rise to speculations having far-reaching cosmological consequences. Sulentic (1984) and Arp and Sulentic (1985) favoured a noncosmological interpretation of the nature of the redshifts of the members of bound groups. Byrd and Valtonen (1985) proposed that the excess of positive redshifts results from most of the group population being composed of unbound expanding members and from a mistaken tendency to pick out a galaxy of brighter apparent magnitude from the nearer portion of the group population rather than the true first-ranked galaxy, which would generally be in the middle of the population.

Examining a subsample of nearby "bona fide" groups (with the radial velocity of the first-ranked member $<1500^{-1}$ ) of the catalogue of Geller and Huchra (1983), we confirm the existence of redshift asymmetry. For the 14 nearby groups with more than 10 observed members, the number of positive velocity differences turns out to be $N+=48$, while the number of negative values $N-=12$, with a ratio $N+/ N-=4$. On the other hand, farther groups show no significant redshift asymmetry (they are less well-known).

\section{INTERPRETATION}

We argue that the redshift asymmetry can be explained by the presence of intragroup extinction (by dust) in bound groups that are in a phase of collapse. In collapsing groups with intergalactic dust, galaxies on the nearer side of the system are redshifted with respect to the center of it and less obscured than those that are blueshifted, which are on the farther side. Since the catalogue of groups is magnitude-limited at $m_{B}=14.5$, their observed number is smaller than that of redshifted members. Assuming a spherical distribution (with constant mass density) of the galaxies of a group and a constant number density of dust grains, we have evaluated the expected distribution of redshifts. The observed redshift asymmetry, $N+/ N=4$, corresponds to a maximum optical depth $\zeta \sim 1$ for a standard value of the group distance $D=15 \mathrm{Mpc}$, a group 
radius $R=0.5 \mathrm{Mpc}$ using the galaxy luminosity function of Geller and Huchra (1983), a typical normalized luminosity of the second-ranked galaxy $l_{2}=L_{2} / L^{*}=0.2$ with $L^{*}$ corresponding to $M^{*}=-19.4$. For typical spherical grains (of graphite or dirty ice) of radius of $0.1 \mu \mathrm{m}$, the density of the intragroup dust is $5.10^{-30} \mathrm{~g} / R\left(\mathrm{~g} / \mathrm{cm}^{3}\right)$, with $\mathrm{R}$ in Mpc. Hence, the density of the intragroup dust turns out to be on the order of $10^{-29} \mathrm{~g} / \mathrm{cm}^{3}$.

As an estimate of the center of mass of a group, we have chosen that indicated by the brightest member, and not the mean velocity of the observed members. It may happen that a galaxy fainter but less obscured than the brightest one is assumed to represent the position of the center of mass; in this case the observed asymmetry is less pronounced than the true one.

If dust is present in a group, one expects that the galaxies on the nearer side of a group, redshifted with respect to the center of mass, should present a colour excess $E(B-V)$ which is smaller than that of the galaxies on the farther, blueshifted side. We have found a significant correlation $E(B-V)$ - (normalized) velocity difference; for 40 galaxies whose colour indices $(B-V)_{0}^{T}$ have been taken from the RC2 catalogue (de Vaucouleurs et al., 1976), we obtained the Spearman correlation coefficient $r=-2.51$, which is significant at the $99.4 \%$ level. We found a similar correlation for the colour excess $E(U-B)$.

The existence of negative correlations between the colour excesses $E(B-V)$ (or $E(U-B)$ ) and the velocity differences can be used to discriminate between our interpretation and the explanation suggested by Byrd and Valtonen (1985). According to their view, one should expect no correlation at all, or if dust is diffuse in space, galaxies with positive velocity differences with respect to the first-ranked member should be farther away than those with negative velocity differences; hence, a positive correlation between colour excess and velocity difference should be expected.

\section{REFERENCES}

Arp, H. and Sulentic, J.W.: 1985, Astrophys. J. 291, 88.

Byrd, G.G. and Valtonen, M.J.: 1985, Astrophys. J. 289, 535.

de Vaucouleurs, G., de Vaucouleurs, A., Corwin, H.: 1976, Second Reference Catalogue of Bright Galaxies (Austin: University of Texas Press).

Geller, M.J. and Huchra, J.: 1983, Astrophys. J. Suppl. 52, 61.

Sulentic, J.W.: 1984, 286, 442. 